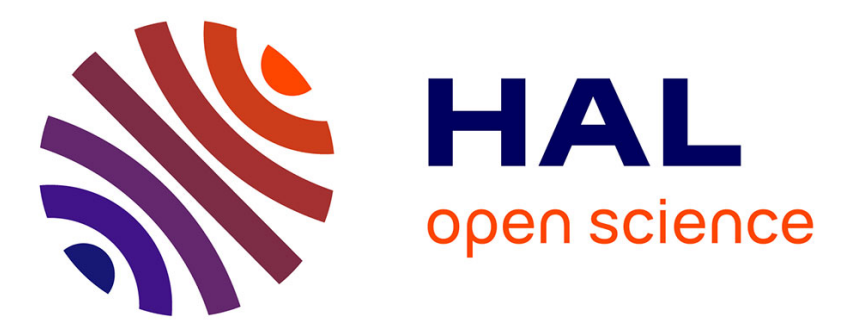

\title{
Influence of vibrotactile feedback on some perceptual features of violins
}

\author{
Indiana Wollman, Claudia Fritz, Jacques Poitevineau
}

\section{To cite this version:}

Indiana Wollman, Claudia Fritz, Jacques Poitevineau. Influence of vibrotactile feedback on some perceptual features of violins. Journal of the Acoustical Society of America, 2014, 136 (2), pp.910-921. 10.1121/1.4889865 . hal-01461743

\section{HAL Id: hal-01461743 \\ https://hal.science/hal-01461743}

Submitted on 1 Sep 2021

HAL is a multi-disciplinary open access archive for the deposit and dissemination of scientific research documents, whether they are published or not. The documents may come from teaching and research institutions in France or abroad, or from public or private research centers.
L'archive ouverte pluridisciplinaire HAL, est destinée au dépôt et à la diffusion de documents scientifiques de niveau recherche, publiés ou non, émanant des établissements d'enseignement et de recherche français ou étrangers, des laboratoires publics ou privés. 


\title{
Influence of vibrotactile feedback on some perceptual features of violins
}

\author{
Indiana Wollman, ${ }^{\text {a) }}$ Claudia Fritz, and Jacques Poitevineau \\ Institut Jean Le Rond d'Alembert, Université Pierre et Marie Curie, and UMR CNRS 7190, 4 place Jussieu, \\ 75005 Paris, France
}

This paper investigated how auditory and vibrotactile feedback information is integrated within the context of violin quality evaluation. Fifteen violinists evaluated three violins on four criteria- "Rich Sound," "Loud and Powerful," "Alive and Responsive," and "Pleasure"-during a perceptual experiment. Violinists first evaluated the violins one at a time under three experimental conditions:(1) playing, (2) listening to it (played by a professional player) in an active way by fingering the score on an isolated neck, (3) same as (2) with vibrotactile feedback provided at the isolated neck. Violinists were then asked to evaluate the violins through pairwise comparisons under condition (3): Each violin was paired with itself while the level of vibrations of the isolated neck was either the original one or divided by two. The first part of the experiment demonstrated that Loud and Powerful judgments were affected by the presence of vibrations given that violins were rated louder in condition (3) than in (2). In the second part, violins were rated more positively with original vibra-tion level at the isolated neck than with half the level, for all criteria but Alive and Responsive. Consistently with sensory interaction, the magnitude of the enhancement remained relatively con-stant across violins.

\section{INTRODUCTION}

Violin playing is multimodal in nature. In addition to the resulting sound, violinists receive visual feedback from their performances as well as vibrotactile feedback, which should be related to the sound produced, through different contact points with the instrument. All these sensory feedbacks enable violinists to exert control over the instrument. We assume that in a playing context, the existence of these complex interactions between the musician and the violin are the basis for the perception of the sound and the "feel" of the instrument. In this regard, as hypothesized by Marshall (1985), "to be accepted by an artist, an instrument must not only sound correct but it must also 'feel' correct." It is still not clear to what the notion of "feel" refers to. Acousticians who invoked the notion of "feel" in violin acoustics literature highly suggested it to be a feeling of vibrations through the left hand (Marshall, 1986; Hutchins, 1985; Woodhouse, 1998). But when violinists are asked what they think about the vibrations of the neck of their violin, their responses are scattered. Most of the time, in first place, they do not understand what the question means. One possible explanation to this confusing reaction is that violinists' attention is focused on violin sound more than on what happens at their left hand, considering the variety of sensory information violinists receive while playing and considering that their ultimate goal is to make music be heard. However, their nervous system tends to merge together the available sensory pieces of information-even if the observer is not fully aware of them-in unique events. This process is commonly known as "multisensory integration" (see Calvert et al., 2004, for a

\footnotetext{
a) Author to whom correspondence should be addressed. Electronic mail: wollman@lam.jussieu.fr
}

review). Thus, the potential audio-tactile interaction in violin playing and perception probably goes mostly unnoticed for violinists.

Von Békésy (1959) highlighted the physiological and functional analogies featuring the relationship between hearing and touch, in particular in terms of vibratory information processing (both senses conveying oscillatory patterns), although he pointed out as well some discrepancies between auditory and tactile systems (e.g., absolute thresholds or frequency ranges of sensitivity associated to each modality). These similarities allow the existence of crossmodal interactions at multiple levels along the functional architecture of the sensory systems. In particular, further research showed the potential for the auditory cortex to process vibrotactile information (e.g., Kayser, 2005). Literature addressing audio-tactile interaction has shown that auditory intensity ratings systematically increased when tones were accompanied by synchronous tactile stimuli compared to when they were presented in isolation (e.g. Gillmeister and Eimer, 2007; Yau et al., 2010). Like for any other sensory integration, audio-tactile integration is stronger for temporally coincident stimuli, stronger for stimuli closer in space, and obeys to the principle of inverse effectiveness-greater enhancement for less effective stimuli-(see Stein and Meredith, 1993).

However, it should be noted that research on multisensory integration has chiefly focused on the perception of spatial and temporal characteristics of multimodal events in laboratory context. The ecological validity of those findings has not received extensive study (Giordano et al., 2010) and only a few studies have tried to address more realistic situations. For instance, it was shown that the perception of the pleasantness and roughness of an electric toothbrush (Zampini et al., 2003) or the crispness and staleness of potato 
chips (Zampini and Spence, 2004) could be affected by the sound of, respectively, the brushing action or the biting action.

Despite Musacchia et al. (2008) pointing out that "musical instruments provide a wide range of naturally occurring complexities to test theories of multisensory interaction," such a musical context has only been rarely used. In the particular case of the perceptual evaluation of instruments, which is our primary interest, Keane and Dodd (2011) investigated a possible improvement of upright pianos by reducing key vibrations. Pianists were invited to play and complete a comparison evaluation task. It was found that pianists felt there were larger changes in tones or loudness between two pianos whose only difference concerned their key vibration level (sounds were measured to be unchanged by the modifications). This result highlights the existence of multimodal interaction in piano playing and evaluation with auditory and tactile feedbacks being combined during their evaluation process.

In the present study, we investigated the role of vibrotactile cues in the perceptual evaluation of violins from both the player's perspective and from the listener's perspective. In the latter case, the listener (violinist) could be provided with vibrotactile information of the violin being heard. One aspect of our experiment was indeed specifically designed to test an "augmented listening" condition, where the listener could receive a vibrotactile feedback of the playing. To this end, we constructed an experimental setup based on the use of an isolated vibrating neck, directly connected to a real violin, which provided the listener with the actual vibration of the neck of the violin being played live by a professional violinist in real time. The isolated neck maximizes ecological validity because the listener has enough cues to mimic a normal playing situation. This setup allows for modulating the level based on a carefully controlled procedure. That way, the two types of signals (audio and tactile) are uncoupled, although they are generated by a common event: the professional violinist drawing his bow across the strings. The use of an isolated neck prevents the excitation of the violin body by the neck and thus the generation of sound which would interfere with the actual evaluation and guarantees that the violinists feel the vibrations through the left hand only.

The perceptual experiment presented in this paper primary aims to explore the role of the level of vibrotactile feedback in modulating the perception of the sound of a violin (maximizing ecologically valid condition). We examined whether the actual experience of sound, expressed by the ratings of a priori auditory-based evaluation criteria, can change as a function of vibrational input, at the left hand. In particular, considering the results of previous studies on auditory perceived intensity, it was hypothesized that we should observe an enhancement of the Loudness and Power ratings of violins when the vibrotactile feedback is provided compared to when it is not, and compared to when it is lower.

Moreover, like Askenfelt and Jansson (1992), we found it interesting to ask whether the vibrations can contribute to the "excitement of playing." We investigated this issue by testing the effect of the vibration displayed along with the sound on the ratings of the Pleasure listeners would have to play the violin being heard. It was hypothesized that increasing the strength of the vibration in the neck would lead to an improvement in subjective pleasure.

Therefore, the violins were evaluated on the criteria Loudness and Power and Pleasure. Two other criteria were chosen. The criterion "Sound Richness" was selected based on the results of our previous experiment (Wollman et al., 2013) where this criterion appeared to be relevant to about a third of the 20 participants while carrying out a violin evaluation task under auditory masking conditions. This criterion was thus suggested not to relate unequivocally to sound properties but to be potentially based on multisensory information processing as well. The last criterion selected was Alive and Responsive. We initially wanted to test the criterion "Alive" because it was stated by Marshall (1986), an acoustician, that violinists prefer instruments that feel alive in the sense of vibrating. However, informal discussions with violinists led us to add the term "Responsive" in order to orient the many and ambiguous definitions of Alive toward the tactile sense. We predicted that if vibrotactile cues affect the perception and evaluation of those two criteria, then our participants should perceive the violin features to vary as the level of vibration feedback was manipulated, whatever the direction of the effect is.

Finally, one open question is to know whether violinists evaluate violins in a different manner when they are successively in the role of the player (i.e., with the natural vibrotactile feedback of the instrument to the left hand, through the neck, as well as to the right hand, through the bow-string interaction), in the role of a simple listener (i.e., no vibrotactile feedback), or in the role of an "augmented listener" (i.e., vibrotactile information provided to the left hand).

We investigated the above questions by asking violinists to rate the selected criteria under the different experimental conditions. The violins used in this experiment were chosen to be sufficiently different in terms of vibratory behavior to see whether the effect of vibrotactile feedback can depend on the violin.

\section{EXPERIMENT}

\section{A. Method}

\section{Participants}

Fifteen classical professional or semi-professional violinists took part in the experiment: six females and nine males, all were naive about the purpose of the study. They ranged in age from 20 to $59 \mathrm{yr}$ old (average age $=35 \mathrm{yr}$, $\mathrm{SD}=10$ ), had played violin for 13-46 yr (average years of violin practice $=27 \mathrm{yr}, \mathrm{SD}=10$ ), played around $30 \mathrm{~h}$ per week on average. Participants reported mainly playing in various contexts [chamber ensembles $(80 \%)$, orchestras (33\%), orchestras as soloist $(20 \%)$ violin classes as professors $(20 \%)$, violin classes as student (20\%)]. None of them reported having auditory or tactile deficits. The violinists were paid for their participation. 


\section{Violins}

A set of 12 violins of different make and age-made between the early 17 th and 21 st centuries-was initially assembled for this study.

To minimize fatigue for the participants, given that several experimental conditions were to be tested, only three violins were included in the experiment. Thus, a preselection test was carried out prior to the experiment. A professional violinist was invited to play freely the set of 12 violins, blindfolded, for evaluation purposes. He was encouraged to comment out loud about the evaluation process and to report orally his feelings regarding the sound and the "feel" of each violin. His comments about how the violins sounded and "felt" and especially, all that was related to the "touch" and his tactile sensations, led us to restrict the study to three instruments that were perceived as the most different in terms of vibratory behavior. However, with only three violins, it cannot be ruled out that participants would focus their attention on identifying the three violins and try to be consistent over conditions, which would distract the participants from the actual task. Consequently, we proceeded as follow: the professional violinist selected three more violins among the remaining ones, chosen to be of similar overall quality to that of the others so that none of these three new violins could stand out and affect the range covered by the previously selected violins. These six violins were presented to the participants during the familiarization phases. To further ensure that participants made judgments using only sensory information, they were specifically instructed that some of those violins would be randomly presented in each condition, whereas, in fact, the same three violins were presented to all participants. These three violins will be referred to as $\mathrm{V} 1, \mathrm{~V} 2$, and $\mathrm{V} 3$.

The fact that some violins may have been less optimally setup or had strings of varying quality was not a concern because it should not have influenced the consistency of the ratings across conditions. Identical shoulder rests (Kun Original model) were used for all six violins. As in the experiment of Saitis et al. (2012), participants performed with their personal bows, which through constant use can be assumed to give the player the best conditions for evaluating an unknown instrument.

\section{Procedure}

The experiment employed a near-blind evaluation task in which participants rated the three violins according to three criteria related to violin attributes and according to the pleasure they have/would have to play the violin. The combination of low lighting conditions and dark sunglasses worn by the participants throughout the conditions was chosen to prevent detailed visual feedback which could constitute irrelevant cue as regards to our research question. The experiment took place in a room of about $30 \mathrm{~m}^{2}$, of relatively dry acoustics. The room was divided into two areas by means of an acoustically transparent curtain. The violinist experimenter was seated on one side of the curtain; the participants occupied the other side of the curtain (see Fig. 1).

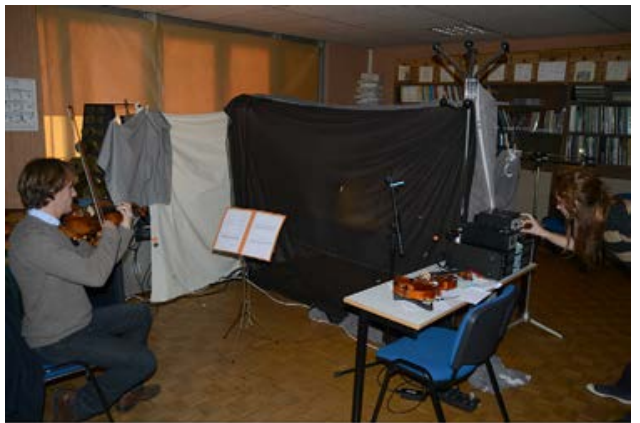

(a)

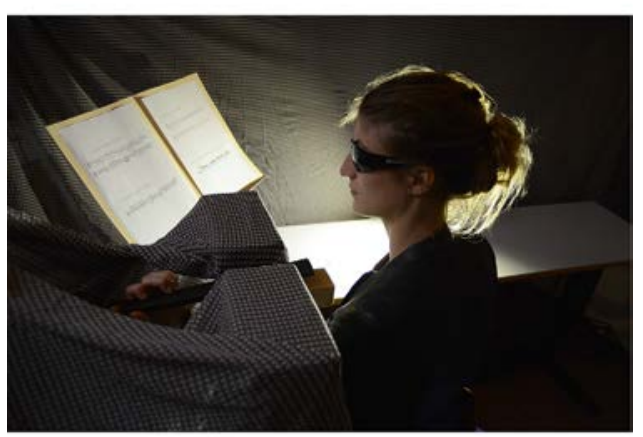

(b)

FIG. 1. (Color online) Experimental room: violinist experimenter on one side of the curtain (on the left), participant holding the isolated neck on the other side of the curtain (on the right).

Prior to the experiment, participants were informed they would evaluate a number of violins in two distinct situations: a playing situation (Situation $\mathrm{P}$ ) and an active listening situation (Situation L).

a. Experimental design. As shown in Fig. 2, there were two experimental factors:

(1) the "situation," describing the participant's role: either player (Situation P) or listener (Situation L);

(2) the "vibration level" in the isolated neck: either normal, like in the violin neck being played (hereafter, "100\%") attenuated, half the level (hereafter, "50\%"), or null (hereafter, " $0 \% ")$.

The second experimental factor applies to the second level of the first factor only.

Thus, there were four experimental conditions:

(1) Playing (Cond P);

(2) Augmented Listening 0\%, also called Listening (hereafter, Cond L);

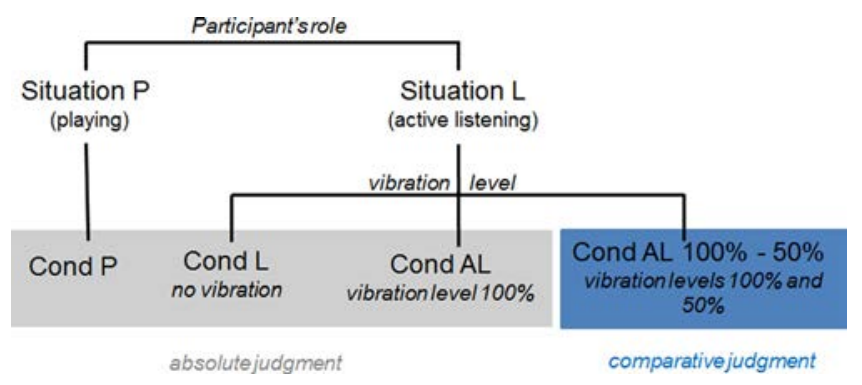

FIG. 2. (Color online) Experimental design. 
(3) Augmented Listening 100\% (hereafter, Cond AL),

(4) Augmented Listening 100\% and 50\% (hereafter, Cond AL 100\%-50\%).

In all four conditions the auditory feedback was normal, in Cond $\mathrm{P}$ as player and in Cond $\mathrm{L}$ and $\mathrm{AL}$ as listener at a close distance. Vibratory feedback was provided as normal to both left and right hands (via the neck and bow) in Cond $\mathrm{P}$, and to the left hand only in Cond AL. In Cond L no vibratory feedback at all was given. All participants completed the evaluation task under the four experimental conditions.

As illustrated in Fig. 2, there were two types of evaluation tasks, either absolute judgment (first three experimental conditions) or comparative judgment (last experimental condition).

In Situation P, participants were invited to play and rate the violins. No constraints were imposed on the repertoire. Imposing violinists to play the same musical excerpts as in the other (listening) conditions (see below) may have appeared as a more controlled situation but it would have been too far from violinists' actual practice, when they test violins (in order to buy one). Consequently, we chose to let them play the instruments freely to maximize ecological validity. In this situation, the participants could choose to play standing up or sitting down.

In Situation L, participants were seated on a chair and held the isolated neck. They were asked to evaluate the violin being played by the violinist-experimenter while actively listening to it. Actively means that they were instructed to hold the isolated neck, and finger on it following the music played by the violinist experimenter, as if they were playing it themselves. One particular musical excerpt (see Sec. II A 3 b) was chosen for each criterion for two main reasons. First, in terms of feasibility, it was necessary for the participants to have a musical score to be able to follow the violinist experimenter in real time. A score compiling the different excerpts was thus placed in front of them. Second, this is close to violinists' actual practice as they usually have to rely on short excerpts when evaluating a violin just by listening to it (for instance when they are asked to advise a friend). In Situation L, the experimental conditions Cond $\mathrm{L}$ and Cond $\mathrm{AL}$ were mixed. The participants thus proceeded with six presentations: the three violins at $0 \%$ and the three violins at $100 \%$. The order of presentation was randomized across participants.

For practical reasons, all participants started with Situation P so we had to mount the violins with the accelerometers only once (for the rest of the experiment).

There were two types of evaluation tasks:

(1) In Situation P and Situation L/Cond L and Cond AL, participants were instructed to complete a series of evaluation task for one violin at a time. For each violin, participants were presented with the evaluation criteria appearing one at a time in random order. They were asked to assess the magnitude of each criterion by placing a marker along a continuous $10-\mathrm{cm}$-long rating scale labeled "not at all" on the left and "a lot" on the right, drawn on a notebook prepared for the experiment. The position of the marker constitutes score between 0 and 10 .
(2) In Situation L/Cond AL 100\%-50\%, participants were instructed to evaluate the violins based on paired comparisons. Three pairs of violin were presented to the participants. Each violin was paired with itself as the level of vibrations set at the isolated neck was either the normal one $(100 \%)$ or divided by two $(50 \%)$. In this way, one pair for violin V1 corresponded to V1_100\% versus V1_50\%. The same applies for the two other pairs, with V2 and V3. For each pair, participants were presented with the evaluation criteria appearing one at a time in random order. For each criterion $X$, the participant answered the question "which violin is more $X$ " by placing a marker along a continuous $10-\mathrm{cm}$-long rating scale ranging from "The first violin is much more $X$ than the second violin" to "The second violin is much more $X$ than the first violin" (the central position thus corresponded to "The first violin is as $X$ as the second violin").

The position of the marker constitutes a score between -5 and +5 . Although the order VL_50\%/VL_100\% was randomized across pairs and participants, the results are hereafter systematically reported so that a positive rating means that the violin with VL_100\% has the attribute $X$ more than the one with VL_50\%.

All participants performed the two tasks in the order listed above.

Participants were specifically instructed not to try to identify the violins because the instruments would be presented randomly. Thus, the actual number involved in the different situations was not disclosed. Participants had 15 min before Situation P to play and freely explore the six violins including the three violins of the actual experiment (see Sec. II A 2) to get an idea of the range covered by the violins under study. In the same way, they had $10 \mathrm{~min}$ before Situation $\mathrm{L}$ to familiarize themselves with the experimental situation by listening to the violinist-experimenter performing on the six violins and on their own violin if they wanted to have a reference. No vibrations were sent to the isolated neck in the familiarization phase.

The individual session lasted approximately one hour and a half.

b. Evaluation criteria and musical excerpts. The four evaluation criteria chosen were presented in the form of a short sentence, as follows:

(1) This violin has a rich sound (in overtones).

(2) This violin is loud and powerful.

(3) This violin is alive and responsive.

(4) Degree of pleasure to play (in situation P)/would have to play (in situation $\mathrm{L}$ ) the violin.

As already mentioned, the criteria were selected on the basis of the results of previous experiments investigating violin quality evaluation. Criteria Rich Sound and Loud and Powerful a priori mainly relate to audition, criterion Alive and Responsive a priori mainly relates to the tactile sense, and criterion "Pleasure" is more related to the overall impression of violinists. Only four criteria were selected to minimize fatigue for the participants, considering the number of experimental conditions to be tested. These terms 
being all commonly used by violinists when evaluating instruments, they were thus left undefined. At the end of the experiment, participants filled out a questionnaire to provide a precise definition of the three criteria related to violin attributes.

The musical excerpts chosen for the criteria evaluation are well-known musical passages of violin repertoire, of duration less than $1 \mathrm{~min}$. They are playable in first position (see technical constraints introduced by the experimental setup in the next section).

(1) Rich Sound (in overtones): G Major scale in thirds

As the "richness" relates to the spectral content of the sound produced, the scale offers the possibility to hear the sound over the register of the violin, across all strings.

(2) Loud and Powerful: Bruch's Violin Concerto No. 1 in G minor, Op. 26, Allegro moderato (second theme)

As the experiment took place in a relatively small room, we assumed it would have been too hard to distinguish the violins with a too loud musical excerpt. The excerpt was thus specifically chosen because of its dynamics $m f$ $(\operatorname{not} f)$.

(3) Alive and Responsive: Bruch's Violin Concerto No. 1 in G minor, Op. 26, Allegro moderato (first theme)

The excerpt was chosen because it goes through all strings and it is not too fast (i.e., not to orient listener particularly toward articulation).

(4) "Degree of Pleasure": Bach Sonata For Violin Solo No 1 in G minor BWV 1001, Adagio (first bars)

The excerpt was chosen because it enables the listener to hear and feel the behavior of this violin across all strings.

c. Violinist-experimenter. One professional violinist (hereafter called FM) was recruited to play the violins for the participants in Situation L. Being a concertmaster of various orchestras, his playing is reliable enough to ensure small variability on the same excerpts over the length of the experiment. Perceived differences were thus likely due to differences between instruments rather than to variability in his playing technique (which would have been impossible to compensate by repetitions, as it would have led to too much fatigue, or obviously by the use of bowing machine). In addition, FM is consultant for a luthier, which means that he is used to test all kinds of violins and to adjust quickly so the violins sound "good," regardless of his personal taste. This means that he is used to adapting to a violin to the extent of making it sound nice but not to the extent of getting his "own" sound (the sound he would try to obtain if he had to play that violin in a concert) in order not to erase subtle differences between instruments.

\section{Apparatus and materials-Experimental setup in the AL condition}

In Situation L, the experimental setup basically consisted of two parts (see Fig. 3):

(1) the excitation system: a real violin played by the violinist-experimenter;

(2) the retransmitting system: an isolated neck bonded by the neck wedge to a massive block of pine wood in arch form that the participants were asked to hold. Two lead weights of $10 \mathrm{~kg}$ each laid on top of the wooden arch, on both sides of the neck and the arch feet were standing in sand to ensure that the structure remains completely still and was uncoupled from the table on which it was positioned.

The violinist-experimenter played a violin to generate the vibrating signals, audio and tactile. As the participants were seated right in front of the violinist-experimenterbehind the curtain - the sound of the violin was heard live. As regards the tactile signals, the vibrations of the violin were picked up at the violin scroll by means of a small accelerometer (Brüel \& Kjaer, type 4374; weight: 0,65 g) held tightly at the back of the scroll with an elastic band and then sent to the isolated neck through a shaker system (Brüel \& Kjaer mini-shaker, type 4810), in real time. The use of a shaker enabled to excite the structure at different vibration levels. The stinger rod of the shaker (stainless steel, rod diameter, $0.6 \mathrm{~mm}$; length, $85 \mathrm{~mm}$ ) was bonded to the scroll so that the vibrations were efficiently transmitted to the isolated neck. The accelerometer detected the vibrations in the vertical direction (along the $z$ axis, i.e., normal to the fingerboard plane); the shaker excited the scroll of the isolated neck in the same direction (along the $z$ axis).

Ideally, we would have liked to measure the vibrations on the real violins and retransmit them on the isolated neck as close as possible to the position of the hand. The first issue was that it was virtually impossible to set up an experimental device which could follow the hand of the violinist experimenter as well as follow the hand of the participant. One position on both the real violins (for recording the vibrations) and the isolated neck (for retransmitting them) had therefore to be chosen and as a consequence, it was not

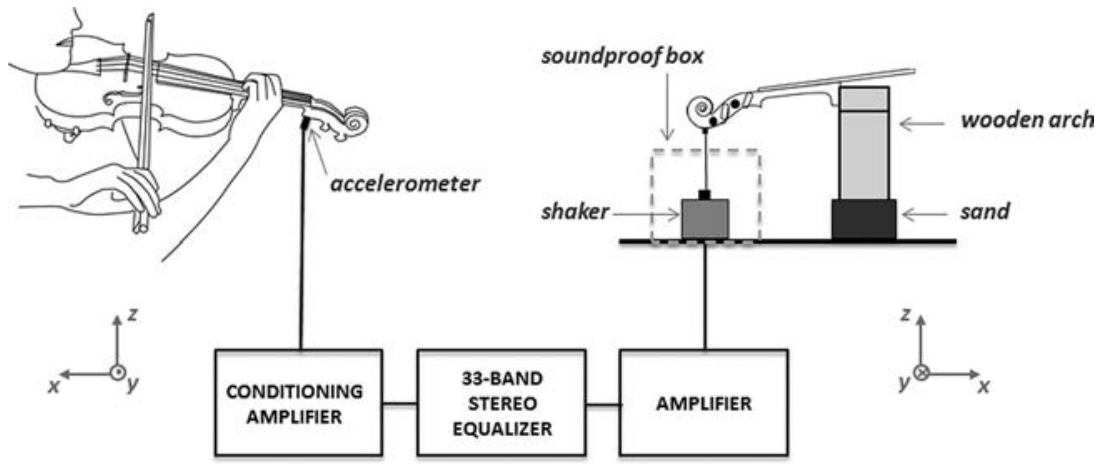

FIG. 3. Experimental setup-the violin coordinate system is represented for both the played violin (left) and the vibrating neck (right). 
possible to recreate the dynamic properties of the real violin neck at all points along the isolated neck. We thus decided to ensure the same vibration level in both necks at a specific location only and we chose the first position. The second issue is that we wanted the participant to hold the isolated neck and the violin-experimenter the real violins as normally as possible. Therefore the shaker and the accelerometer could not be positioned exactly where the hand is in first position, but at the scroll. This can however be compensated for, as explained below in Sec. II A 4 a.

An amplification system was included in the setup (see Fig. 3): a conditioning amplifier (Bruel \& Kjaer 2692D NEXUS) for the accelerometer, a 33-band stereo graphic equalizer (Technics SH-8065) and an amplifier (Bruel \& Kjaer type 2706) for the shaker. The apparatus was concealed behind a curtain.

a. Calibration of the system. Our major concern was to determine the excitation level of the shaker so the resulting vibration felt by the participant's hand holding the isolated neck was equal to what was felt by the violinistexperimenter's hand at the real neck.

We assumed that at frequencies higher than $50 \mathrm{~Hz}$, which is clearly in the vibrotactile domain, people are not sensitive to the direction of the vibrations of the object in contact with their hands; they feel the vibration as a whole. Although to our knowledge there is no behavioral study investigating this assumption, there is a physiological basis to support it. The Pacinian receptor population is likely to provide the bulk of the neural signaling in this frequency range (Lamoré et al., 1986), and this population is distributed in deeper tissues (Stark, 1998), where the effect of the boundary conditions can be expected to have vanished.

As a result, we decided to quantify the strength of the vibrations of both the real and isolated necks through the root-sum-of-squares (RSS) of acceleration signals recorded in three directions at the player's thumb ( $x$-direction, tangential to the neck; $z$-direction, normal to the fingerboard; and $y$-direction, normal to the contact areas on the sides of the neck, see Fig. 3). The squared RSS value is proportional to the total vibrational energy of the neck. Considering this, our only concern was to make the RSS accelerations of the isolated and real necks equal. We could thus reformulate our problem to take into account this physiological assumption as follows. We needed to compensate the excitation of the shaker so the norm of the resulting vibrations in the isolated neck along the three dimensions was equal to the norm of the vibrations in the real neck.

To this end, it was necessary to calculate some relevant transfer functions, to estimate the overall effect of the chain. Since the input to the chain was provided by vertical acceleration $a_{p s, z}$ measured at the scroll of the played violin (index $p s$ ) and not directly by the RSS acceleration $A_{p h}$ at the position of the hand (index $p h$ ), it was necessary to define the transfer function between the hand and the 1Daccelerometer at the scroll in order to compensate for the "shift" in position as well as the reduction from three dimensions to one. The same applied to the isolated neck where we needed to compensate for the "shift" in position of the shaker, compared to the location of the participant's hand as well as to compensate for the fact that though the excitation was in one direction only (vertical, $z$ axis), vibrations along the $x$ and $y$ axes were produced as well at the position of the participant's hand. And finally, the frequency response of the shaker had to be taken into account too.

Therefore, we first measured the three transfer functions $G_{z x}, G_{z y}$, and $G_{z z}$ between the $z$-acceleration at the scroll of the played violin and the acceleration at the contact between the thumb of the violinist's left hand and the violin neck along the three dimensions and combined them so we got a "transfer function in norm" $G_{\text {norm }}=\sqrt{G_{z x}^{2}+G_{z y}^{2}+G_{z z}^{2}}$, where $G_{z x}=a_{p h, x} / a_{p s, z}, G_{z y}=a_{p h, y} / a_{p s, z}, G_{z z}=a_{p h, z} / a_{p s, z}$. The accelerations in the three directions from the 3Daccelerometer are indicated $a_{p h, x}, a_{p h, y}$, and $a_{p h, z}$, respectively.

As the flesh of the hand can damp the vibrations, the measurements were conducted on the three violins held by the violinist experimenter in first position so $G_{\text {norm }}$ takes into account the damping caused by the hand during the playing. In practice, we measured the three transfer functions $G$ by tapping the back of the scroll with a mini-force hammer (PCB, Model 086E80) next to the 1D-accelerometer (Brüel \& Kjaer, type 4374) in the direction of the accelerometer's axis ( $z$ axis) (see Fig. 3). The response was measured in the three directions by means of a 3D-accelerometer (Brüel \& Kjaer triaxial Deltatron ${ }^{\circledR}$, type 4525-B-001, weight: $6 \mathrm{~g}$ ) mounted on the neck next to the thumb of the violinist. The hammer was thus only used for excitation purposes: the input of the transfer function was not the hammer signal but the 1D-accelerometer response. The 1D-accelerometer remained at the same position during all the experiments.

Second, for the isolated neck (index $i$ ), we measured the three transfer functions $H_{z x}, H_{z y}$, and $H_{z z}$ between the input voltage $U$ to the shaker and the acceleration along the three directions at the place where the participant's thumb would be in first position $\left(a_{i, x}, a_{i, y}, a_{i, z}\right)$ and combined them in a

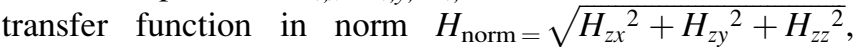
where $H_{z x}=a_{i h, x} / U, H_{z y}=a_{i h, y} / U, H_{z z}=a_{i h, z} / U$. In practice, the three transfer functions $H$ were measured by supplying the shaker with a sweep voltage of constant amplitude and measuring the vibrations of the isolated neck with the same 3D-accelerometer used for the transfer functions $G$, placed where the participant's thumb would be in first position. It was not measured with the participants' hand on the neck for two reasons. First, it would have been impossible to measure it and then adjust the total gain of the chain $B$ (see below) for each participant prior to the experiment. Second, the influence of the hand on the vibrations of the isolated neck was checked to be negligible. Indeed, though the boundary conditions of the neck (clamped by neck wedge to a fixture) are different when held and free, a finite element model of the isolated neck blocked at the neck wedge showed that the eigenmodes of vibration in both configurations are extremely similar, the shift in frequency being only significant at the fourth decimal.

Let $A_{p h}$ be the RSS acceleration at the violin neck of the played violin that we would like to recreate at the position of the participant's hand on the isolated neck. On the played 

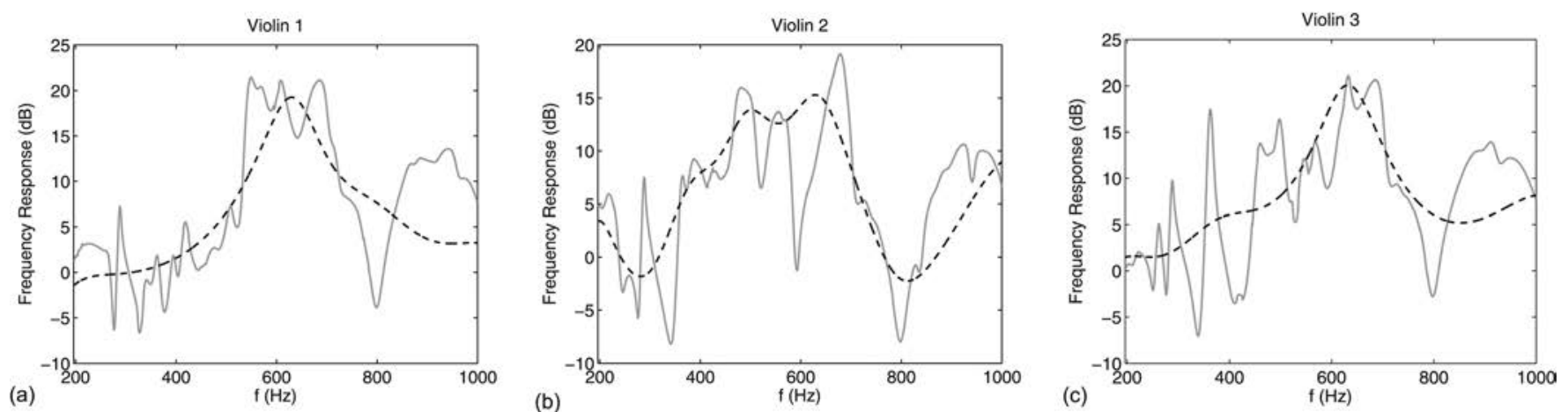

FIG. 4. Overall transfer function $B$ superimposed with the response of the equalizer, for the three violins.

violin, the $z$-acceleration picked up by the accelerometer at the scroll is $a_{p s, z}=A_{p h} / G_{\text {norm }}$. Therefore, the gain $B$ to apply to the input voltage $U$ of the shaker so that we get $A_{p h}$ at the position of the participant's hand on the isolated neck is $B=C \times G_{\text {norm }} \times\left(1 / H_{\text {norm }}\right)$. The quantity $C$ represents the amplification introduced by the amplifier connected to the shaker. $C$ could be 1 (in Cond AL 100\% absolute and comparative judgment) or 0.5 (Cond $\mathrm{AL} 50 \%$ comparative judgment).

An equalizer was thus used to obtain the correct gain $B$. Since the equalizer was based on a bank of filters covering the vibrotactile spectrum in eight frequency bands, each of which having a fixed center frequency and Q but an adjustable level, we could raise or lower each slider in order to visually approximate the graph of the intended frequency response. Our concern was thus to keep the same shape by averaging the signal per frequency band (because it could not be more optimally setup due to the fixed center frequency of the filters). Anyway, $B$ presenting sharp peaks and troughs, it would have not been possible to recreate exactly $B$ by a more sophisticated digital equalizer without introducing delays.

Figure 4 presents, for each violin, the overall transfer function $B$ superimposed with the response of the equalizer as set up to approximate optimally $B$.

The fact that the equalizer only approximated the overall transfer function was not a concern because we did not intend to characterize our violins; the violin is treated as a fixed effect in our experimental design. What was important however was to have realistic levels of vibrations over the range of sensitivity to vibrations. ${ }^{1}$

b. Perceptual validation of the soundproof box. The last issue we encountered was the noise generated by the shaker which acted as a loudspeaker. A soundproof box was specially designed to house the shaker in order to attenuate as much as possible the sound that could emanate from the device and may taint the results. The wooden attenuation box contained a very thin aperture through which the shaker rod could pass.

Preliminary measurements with microphones displayed a very slight increase of the sound pressure level in the frequency band around $1100 \mathrm{~Hz}$ when the setup was switched on. Thus, in order to test the efficiency of the soundproof box on a perceptual level, i.e., whether or not a sound could be heard when the setup was switched on, a test employing a three alternative forced choice (3-AFC) procedure was then carried out. Seven subjects participated in this preliminary test.

The violinist experimenter was asked to play a scale from $\mathrm{G} 5(784 \mathrm{~Hz})$ to $\mathrm{G} 6(1568 \mathrm{~Hz})$ at a dynamic forte, on one of the three violins of the experiment. The vibration transmission setup could be switched on or off. The subjects were seated where participants would sit during the experiment, so their ears were at the correct distance from the setup. As the subjects did not hold the isolated neck, no vibrotactile cues could be used.

Three scales were played to the subject on each trial, two of them with the setup off, one with the setup on. The subjects were instructed to identify aurally the different condition from each triplet presented. If they were not sure then they must guess. The test consisted of three trials.

The subjects did not receive any information other than the instructions and a specification of the sensory modality - audition - by which the difference should be perceived. No information were given about the composition of the three stimulus set with which a subject was presented in a certain trial.

None of the participants gave a single correct answer. Furthermore, all of them reported they picked one of the three options randomly. We thus assumed that noise emanating from the shaker could not be discerned by the participants.

\section{RESULTS}

\section{A. Absolute judgments}

Figure 5 presents the mean criteria ratings (averaged over the three violins) by criterion and experimental condition, in the tasks requiring absolute judgments.

The investigation tested the effect of two experimental factors, the participant's role and the vibration level at the neck. The same overall trend is observed on Fig. 5 for all four criteria. Ratings are higher when participants are in the role of the listener (Cond $\mathrm{L}$ and Cond $\mathrm{AL}$ ) than when they are in the role of the player (Cond $\mathrm{P}$ ). Clear rating increases are indeed observed from the experimental condition Cond $\mathrm{P}$ to Cond $\mathrm{L}$, and then to Cond AL. Mean ratings are around five in Cond $\mathrm{P}$ (on a 0-10 scale), between five and six in Cond $\mathrm{L}$ and slightly above six in Cond AL. The magnitude 


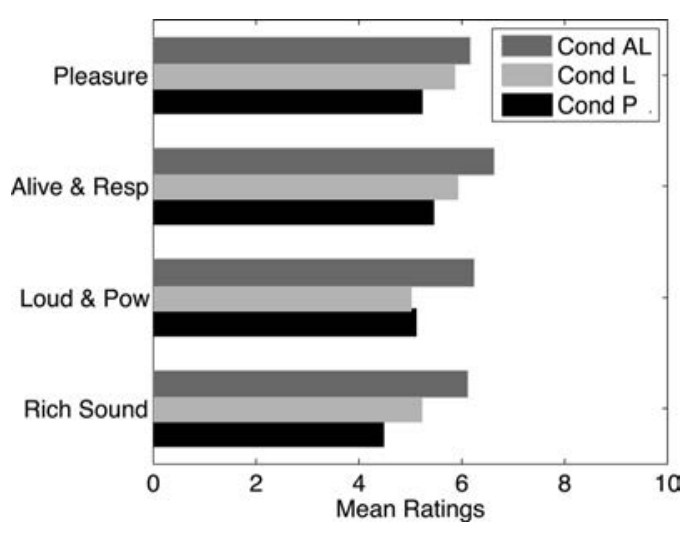

FIG. 5. Mean preference ratings under the three conditions requiring absolute judgments.

of the increase however seems to depend on the criterion (in particular, this has to be qualified for the criterion Loud and Powerful).

Figure 5 shows that when the participants were in the role of the listeners, the simultaneous presentation of vibrotactile information led to an increase in the mean criteria ratings, compared to when no vibrotactile feedback was provided. As a complement of Fig. 5, Table I presents the difference between Cond $\mathrm{AL}$ and Cond L, by criterion. Hereafter, observed one degree of freedom effect of a factor is notated e (e.g. for the main factor "vibration level" Cond AL-Cond L), while the corresponding true effect is notated $\varepsilon$. Bayesian statistical procedures are adopted in this study (Rouanet et al., 2000), using PAC software (Lecoutre and Poitevineau, 1992), ${ }^{2}$ assuming an uninformative prior distribution (i.e., no information is used other than what is contained in the data, no particular hypothesis is favored a priori). Hereafter, Bayesian statements will be notated $\mathrm{Pr}^{*}{ }^{3}$ For instance, in Table I, the last column stands for the 95\% credibility interval: given the data, there is a $95 \%$ probability that the true effect is between -0.22 and 2.01 for criterion Rich Sound.

The observed differences are all positive, and rather small for all criteria. The greatest effect, though limited, is observed for the criterion Loud and Powerful, for which the effect is significant $(p=0.003)$. For this criterion, the same conclusion can be inferred at the population level with sufficient confidence: the upper $\varepsilon$ limit at a $95 \%$ level is only around 2 (compared to the scale $[-10 ; 10]$ ). This demonstrates that adding the vibrotactile feedback information to the listener (violinist) have a clear effect on the evaluation of

TABLE I. Comparisons Cond AL-Cond L.

\begin{tabular}{lcccc}
\hline \hline & $\mathrm{e}(\mathrm{SEM})$ & $\mathrm{t}_{(14)}$ & $p$ & $\operatorname{Pr}^{*}[\mathrm{x} 1<\varepsilon<\mathrm{x} 2]=0.95$ \\
\hline Pleasure & $0.30(0.50)$ & 0.61 & $0.28^{\mathrm{a}}$ & {$[-0.76 ; 1.36]$} \\
Alive \& Resp & $0.71(0.45)$ & 1.56 & $0.14^{\mathrm{b}}$ & {$[-2.66 ; 1.68]$} \\
Loud \& Pow & $1.23(0.38)$ & 3.20 & $0.003^{\mathrm{a}}$ & {$[0.40 ; 2.05]$} \\
Rich Sound & $0.90(0.52)$ & 1.72 & $0.11^{\mathrm{b}}$ & {$[-0.22 ; 2.01]$} \\
\hline \hline
\end{tabular}

${ }^{\mathrm{a}}$ One sided.

${ }^{\mathrm{b}}$ Two sided, according to our hypotheses reported at the end of the introduction.
TABLE II. Comparisons Cond L-Cond P.

\begin{tabular}{lrrcc}
\hline \hline & $\mathrm{e}(\mathrm{SEM})$ & $\mathrm{t}_{(14)}$ & two sided $p$ & $\operatorname{Pr}^{*}[\mathrm{x} 1<\varepsilon<\mathrm{x} 2]=0.95$ \\
\hline Pleasure & $0.63(0.61)$ & 1.03 & 0.32 & {$[-0.69 ; 1.95]$} \\
Alive \& Resp & $0.47(0.40)$ & 1.16 & 0.26 & {$[-0.40 ; 1.33]$} \\
Loud \& Pow & $-0.10(0.50)$ & -0.20 & 0.85 & {$[-1.15 ; 0.96]$} \\
Rich Sound & $0.74(0.53)$ & 1.38 & 0.19 & {$[-0.41 ; 1.89]$} \\
\hline \hline
\end{tabular}

the criterion Loud and Powerful, though this effect is small. This is in line with our hypothesis that our participants should perceive the violin more powerful with the vibration feedback than without, while listening.

Moreover, in this task requiring absolute judgments, our hypothesis concerning the rating of criterion Pleasure is not confirmed nor invalidated because of too much uncertainty, as illustrated by the credibility interval. Again, for the two other criteria, there is too much variability to make any conclusion. So the questions concerning whether or not the vibrations have an effect on their evaluation remain open.

To explore whether violinists evaluate violins in a different manner when they are in the role of the player, in the role of a simple listener, and in the role of an "augmented listener" (i.e., where Cond P is situated compared to Cond L and $\mathrm{AL}$ ), we compared Cond $\mathrm{P}$ and Cond L (Table II) and Cond P and Cond AL (Table III), ${ }^{4}$ by criterion.

In Table II, the observed differences are small for all criteria, and not significant. The null hypothesis that the mean population ratings in both conditions are identical cannot be rejected. Furthermore, all credibility intervals are rather small so it could be concluded with sufficient guarantee that if any of these effects exist, it is not large. In Table III, the observed differences are rather small though quite significant, except for criterion Pleasure [for which $\left.\operatorname{Pr}^{*}(\varepsilon>0)=1-\mathrm{p} / 2=0.97\right]$.

Therefore, while it is not possible to draw conclusion as to the position of the ratings in Cond $\mathrm{P}$ compared to those in Cond $\mathrm{L}$, we showed that with our setup, ratings in Cond $\mathrm{P}$ are below those in Cond AL.

It is interesting to investigate whether the effect of vibrotactile feedback (i.e., the effect of the experimental conditions) is dependent on the violin. Table IV presents the effects of interaction condition $\times$ violin, by criterion (measured as the root mean square of the effects of the four contrasts forming the interaction, and notated root mean square (RMS) for the observed effects and RMS for the true effects).

As can be seen from the table, there was no significant interaction between conditions and violins, so the null hypothesis that whatever the violin, the condition has the same

TABLE III. Comparisons Cond AL-Cond P.

\begin{tabular}{lcccc}
\hline \hline & $\mathrm{E}(\mathrm{SEM})$ & $\mathrm{t}_{(14)}$ & two sided $p$ & $\operatorname{Pr} *[\mathrm{x} 1<\varepsilon<\mathrm{x} 2]=0.95$ \\
\hline Pleasure & $0.94(0.45)$ & 2.07 & 0.06 & {$[-0.03 ; 1.90]$} \\
Alive \& Resp & $1.18(0.37)$ & 3.14 & 0.007 & {$[0.37 ; 1.98]$} \\
Loud \& Pow & $1.13(0.36)$ & 3.13 & 0.007 & {$[0.36 ; 1.90]$} \\
Rich Sound & $1.64(0.48)$ & 3.41 & 0.004 & {$[0.61 ; 2.66]$} \\
\hline \hline
\end{tabular}


TABLE IV. Interaction effects (condition $\times$ violin).

\begin{tabular}{lcccc}
\hline \hline & RMS (SEM) & $\mathrm{F}_{(4,56)}{ }^{\mathrm{a}}$ & two sided $p$ & $\operatorname{Pr}^{*}[R M S<\mathrm{x}]=0.95$ \\
\hline Pleasure & $0.95(0.93)$ & 1.04 & 0.39 & $\mathrm{x}=1.98$ \\
Alive \& Resp & $0.38(0.87)$ & 0.19 & 0.94 & $\mathrm{x}=1.50$ \\
Loud \& Pow & $1.05(1.02)$ & 1.09 & 0.37 & $\mathrm{x}=2.17$ \\
Rich Sound & $1.18(0.86)$ & 1.86 & 0.13 & $\mathrm{x}=2.08$ \\
\hline \hline
\end{tabular}

${ }^{\mathrm{a}}$ Greenhouse-Geisser and Huynh-Feldt corrections for violation of sphericity are negligible.

effect cannot be rejected. Moreover, the highest observed interaction effect is 1.18 (criterion Rich Sound), and the highest limit of the negligibility interval being 2.17 considered as a small value compared to the scale [0 20], we can conclude that the interaction effects, if they exist, are limited.

Hence, even though the violins were chosen to be as different as possible in terms of vibratory behavior, it is still possible that the effect of vibrotactile feedback is independent of the violin.

\section{B. Comparative judgments}

With the paired comparison evaluation we test the hypotheses that, in the augmented listening condition, increasing the vibration level at the neck leads to an increase of the Loud and Powerful and Pleasure ratings. We also explored whether the ratings of criteria Rich Sound and Alive and Responsive varied as the level of vibration feedback was manipulated.

We first study the effect of the factor "level of vibration," violin by violin. Because each pair consisted of the same violin with two different levels of vibration, in the following analyses, we will talk about "effect of violin" rather than "effect of pair." Figure 6 shows the mean comparison ratings for each violin and criterion (a positive score indicates that the violin VL_100\% has the attribute $X$ more than the one with VL_50\%).

Excepted two violins for the criterion Alive and Responsive, the effect of the level of vibration at the neck is clear and quite large on all violins and criteria: the ratings being positive for three criteria, participants tended to rate the violin with $100 \%$ vibrations higher than when the vibration level was $50 \%$.

Table $\mathrm{V}$ presents the main effect of the factor "vibration level" (directly given by the mean difference ratings), e is the observed effect and $\varepsilon$ the true effect.

The effect of the vibration level on the ratings of criteria Rich Sound, Loud and Powerful, and Pleasure is highly

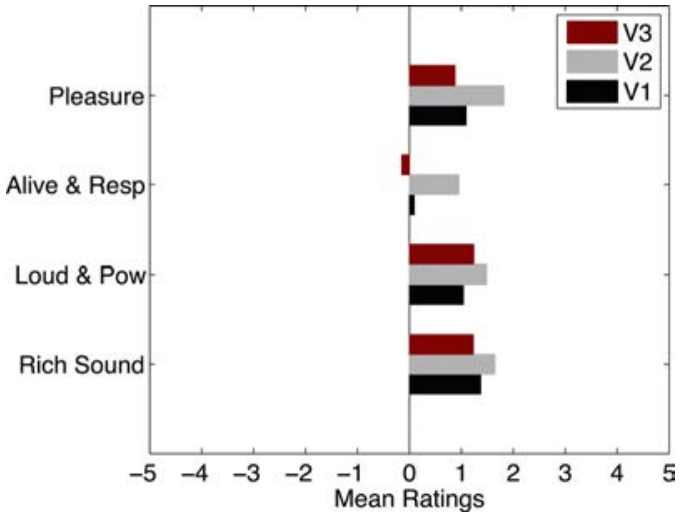

FIG. 6. (Color online) Mean comparison ratings (VL_100\% - VL_50\%) of the three violins, by criterion.

significant $(p<0.0001)$. For these three criteria, the effect is of high magnitude (compared to a $[-5 ; 5]$ scale), and it is the greatest for criterion Rich Sound (1.42). Given the data, the probability that the true (population) effect will be positivei.e. the higher the vibration amplitude, the better the rating-is extremely high for these three criteria, and, moreover, still very high for an effect higher than 1 , that we consider as a quite substantial difference value on a $[-5 ; 5]$ scale.

As regards the criterion Alive and Responsive, the effect is small and non significant. Although positive, the possibility of a negative (or null) population difference cannot be ruled out with sufficient confidence to infer the same conclusion at the population level, so our judgment will be suspended.

Again, we tested whether the effect of the level of vibrotactile feedback is dependent on the violin. As can be seen from Fig. 6, the same overall trend is observed for all three violins. Table VI presents the effect of the factor violin, measured as the RMS of the pairwise mean differences (to be compared to a scale $[0 ; 10])$.

The observed effect of the factor violin is of limited magnitude for all four criteria ( 0.81 at a maximum compared to the scale $[0 ; 10])$ and non-significant. The same conclusion can be inferred at the population level with sufficient confidence as the highest upper RMS limit (at a 95\% level) is 1.62 (obtained for the criterion Alive and Responsive). Consequently, the effect of the vibration level does not appear to depend too much on the violin.

\section{DISCUSSION AND CONCLUSIONS}

This research has investigated the effect of vibrotactile feedback in the left hand of violinist on the perception and evaluation of violin, taking as a starting point some

TABLE V. Effect of the factor "vibration level."

\begin{tabular}{|c|c|c|c|c|c|c|}
\hline & e (SEM) & $t_{(14)}$ & $p$ & $\operatorname{Pr}^{*}[\mathrm{x} 1<\varepsilon<\mathrm{x} 2]=0.95$ & $\operatorname{Pr}^{*}[\varepsilon>0]$ & $\operatorname{Pr}^{*}[\varepsilon>1]$ \\
\hline Pleasure & $1.26(0.20)$ & 6.31 & $<0.0001^{\mathrm{a}}$ & {$[0.83 ; 1.69]$} & $>0.9999$ & 0.90 \\
\hline Alive \& Resp & $0.30(0.29)$ & 1.05 & $0.31^{\mathrm{b}}$ & {$[-0.31 ; 0.92]$} & 0.85 & - \\
\hline Loud \& Pow & $1.25(0.22)$ & 5.79 & $<0.0001^{\mathrm{a}}$ & {$[0.79 ; 1.71]$} & $>0.9999$ & 0.87 \\
\hline Rich Sound & $1.42(0.22)$ & 6.40 & $<0.0001^{\mathrm{b}}$ & {$[0.94 ; 1.89]$} & $>0.9999$ & 0.96 \\
\hline
\end{tabular}

a One sided.

${ }^{\mathrm{b}}$ Two sided, according to our hypotheses reported at the end of the introduction. 
TABLE VI. Effect of the factor "violin."

\begin{tabular}{lcccc}
\hline \hline & RMS (SEM) & $\mathrm{F}_{(2,28)}{ }^{\mathrm{a}}$ & two sided $p$ & $\operatorname{Pr}^{*}[\mathrm{RMS}<\mathrm{x}]=0.95$ \\
\hline Pleasure & $0.70(0.43)$ & 2.65 & 0.09 & $\mathrm{x}=1.27$ \\
Alive \& Resp & $0.81(0.60)$ & 1.83 & 0.18 & $\mathrm{x}=1.62$ \\
Loud \& Pow & $0.32(0.63)$ & 0.26 & 0.78 & $\mathrm{x}=1.26$ \\
Rich Sound & $0.30(0.57)$ & 0.28 & 0.76 & $\mathrm{x}=1.14$ \\
\hline \hline
\end{tabular}

${ }^{\mathrm{a}}$ Greenhouse-Geisser and Huynh-Feldt corrections for violation of sphericity are negligible.

hypotheses generated by researchers in violin acoustics literature and in the field of multimodal perception. On the one hand, in the acoustics domain, the few studies invoking the notion of feel of a violin share a focus primarily on violin necks' vibrations, suggesting that when perceived through the left hand of violinists these vibrations become the basis for the perception of how a violin feels. On the other hand, in the perceptual domain, there are several laboratory demonstrations of tactile influence on the perception of sound. Besides, results of our previous study investigating the role of auditory and tactile modalities in violin quality evaluation (Wollman et al., 2013) pointed to the possible multisensory processes that are likely to occur during the evaluation process of both the feel and the sound of a violin. The present contribution explores whether vibrations can modulate the auditory perception of a violin in approaching realistic conditions. It furthermore constitutes new data on the role of vibrotactile feedback in violin evaluation. We conducted an empirical study in which 15 professional violinists evaluated three violins either by playing them or by actively listening to them while holding and fingering on a real isolated violin neck, to mimic normal conditions. The first part of this perceptual experiment was dedicated to absolute judgments. The second part of the experiment investigated the effect of the level of vibration on the comparative evaluation of two violins of identical sound quality, given that it was the same physical instrument.

\section{A. Loud and powerful}

Our first hypothesis was about the perception and evaluation of the criterion Loud and Powerful in the listening situation. We predicted that the ratings should be higher when violins are evaluated with higher vibrations. In the first part of the experiment (dedicated to absolute judgments), we found that the presence of vibration accompanying the sound of violins had a positive and clear-though small-effect on the evaluation of criterion Loud and Powerful compared to when no vibrotactile feedback was provided. Since there was at most limited interaction between violin and condition, these results stand for all three violins under study.

In the second part of the experiment (dedicated to comparison judgments), we found that a given violin was rated louder and more powerful when accompanied by the actual level of vibrations than when presented with half the vibration level. Again, no effect of violin was found, suggesting that the phenomenon could be independent of the violin. Our results thus confirm our first hypothesis. They provide as well quantitative and perceptual support to previous reports concerning the influence of vibration on auditory intensity perception in laboratory context and, more importantly, extend those findings to the perception and evaluation of musical instrument, in more natural context.

\section{B. Rich sound}

In the task requiring absolute judgments, the effect of the presence/absence of the vibration at the neck failed to reach significance for criterion Rich Sound. Nevertheless, in the comparison task where participants were always provided with a vibrotactile feedback, the level of vibration (full/half) had a strong effect on the ratings of this criterion, namely the higher the vibration amplitude, the richer the sound. Furthermore, the vibrotactile modulation of auditory perception proved to be rather robust because the enhancement is more or less uniform across the three violins. This result is another example of vibrotactile influence on the perception of sound and can be interpreted as support for the existence of crossmodal interaction in violin evaluation.

As regards these two criteria a priori related to auditory-only cues, results in the paired comparison evaluation task show that even though the sound of the violin did not actually change, violinists could not ignore the tactile information. Data quite clearly show cross-modal enhancement, in that participants on average rated the violins as louder and their sounds as richer, when presented with higher vibration than when they were presented with half the level, and this, irrespective of the violin and thus the type of sound.

\section{Pleasure}

A similar trend as seen for Rich Sound ratings was observed for the ratings of criterion Pleasure. Our hypothesis concerning this criterion could not be confirmed, nor invalidated, in the task requiring absolute judgments. It was however totally confirmed in the paired comparison task as expressed by the extremely high probability that the difference VL_100\% - VL_50\% is higher than 1 on a $[-5 ; 5]$ axis. Eventually, our study gives tentative supports to the view that increasing the strength of vibration increases the subjective pleasure. The difference of results in the two parts of the experiment may be explained by the fact that the two types of tasks were extremely different to the participants. The second task being a more direct way of comparing the violins, it may have emphasized small differences (otherwise difficult to evaluate in an absolute way) and thus led to a larger influence of the vibration level (Hsee et al., 1999).

\section{Alive and responsive}

Interestingly, we did not observe any significant effect of the level of vibrotactile feedback on the ratings of criterion Alive and Responsive, neither in absolute judgments, nor comparative judgments. Participants were not instructed before the experiment as to what was meant by Alive and Responsive but were asked to give a definition for each criterion at the end of the experiment and explain how they 
evaluated the criterion during the session. Participants' responses give a plausible explanation to the nonsignificance of the effect on this criterion. One frequent report was indeed that an Alive and Responsive violin is one on which the action of the bow is easy. Now, cues related to bow pressure and articulation, essential for the evaluation of how a violin responses, are not related to the vibration level at the left hand but more to the response of the bow when touching the string as felt in the right hand. Thus, while we added Responsive to orient players toward the most tactile meaning of Alive, with "tactile" in the sense of "vibrotactile at the left hand," it seems that it was interpreted as "tactile as felt in the right hand" which can thus explain this nonsignificant effect of the vibrotactile feedback on this criterion. Following this reasoning, this criterion eventually proves to be useful for control purpose. Considering the participants' definition of the criterion, the absence of an effect for this criterion seems indeed consistent with a sensory effect for the other criteria. If the ratings of Alive and Responsive had increased with increasing vibration level, we could have interpreted the responses of the experiment as the likely result of a decision process (e.g., because of a distraction effect produced by the set up that would result in a shift of attention away from audition and toward the tactile modality). As it is not the case, we can interpret the positive effects on the other three criteria (Loud and Powerful, Rich Sound, and Pleasure) observed in this part of the experiment and reported above as truly reflecting an audio-tactile integration. Incidentally, subjects' comments on the Alive and Responsive aspects of a violin suggested that the vibrations conveyed to the right hand via the bow are important for evaluating a violin. These vibrations give indeed momentary information about the magnitude and status of the bowstring interaction (stable or beginning to break down), and how the instrument responds to changes in bow force and velocity ("ease of bowing"). We did not test whether simultaneity constitutes a necessary factor for an audio-tactile integration to occur-as it is classically done to rule out the alternative distraction account of the results-because violinists are used to combine those two sensory modalities even without being conscious of it, and so we did not want to distract participants toward vibrations. Naturally, it would be premature to conclude that the quality of a violin is necessarily highly correlated to the level of vibration and that large amplitudes of vibration would guarantee the quality of a violin in the violinist community. But these results show a trend for the evaluation to be better for three criteria when, for a given sound, the violin has higher vibrations.

\section{E. Players vs listeners}

One open question raised in the introduction was to investigate whether violinists evaluated violins in a different manner when they play and when they listen. This aspect was addressed in the first part of the experiment dedicated to absolute judgments. Criteria were observed to be more highly rated in the listening situation (Situation L-Cond $\mathrm{L} /$ Cond AL) than in the playing situation (Situation $\mathrm{P}-\mathrm{Cond}$ $\mathrm{P})$, even though no firm conclusion could be asserted for the increase between Cond $\mathrm{P}$ and Cond L. There are several possible explanations to account for this increase. One could argue that this is simply due to an adaptation/habituation effect to the instrument which makes that naturally, the more the violins are heard, the better they are perceived. As all participants started with Situation $\mathrm{P}$, ratings in Situation $\mathrm{L}$ are higher. It could be due as well to the difference of context in which violins were evaluated in those two situations. Participants could freely test the violins in the playing condition but they were constrained in the subsequent listening task. Players could thus use as many techniques as needed and explore as many aspects of violins as wanted to make their judgments in Situation $P$ while they could hear only one musical passage to make their evaluation of the corresponding criterion in Situation L, which likely led to the violins being judged more severely in Situation P (lower marks in average). Furthermore, players can put the amount of sound and vibrations they get in relation to how much effort they feed in, in other words playing exposures how much you need to pay, whereas such a "normalization" is not possible for a listener. Incidentally, one could also argue that, in Cond AL, the vibrational reproduction was not accurate enough - the equalizer only approximated the intended frequency response per frequency band-to allow for comparisons between playing context and augmented listening context. (Note that if it was the case, the limitation of the technique was not a concern in the paired comparison task because we consider only relative judgments.) Although the rather poor frequency discrimination of the tactile sense makes this explanation rather unlikely, it cannot be ruled out that very sharp peaks in the frequency response (not reproduced with our setup) are influential in the overall impression of the instrument. First impression "good notes" of a violin may indeed last long, even if they make the tactile response uneven, and this could only apply to Cond $\mathrm{P}$.

The few studies dealing with sensory integration in a relatively realistic context were always conducted the same way: the audio stimuli that result from an action generating simultaneously a tactile sensation [e.g., teeth brushing (Zampini et al., 2003)] were manipulated in real time to investigate the effect on that tactile sensation. It is therefore the first study, to the best authors' knowledge, that addresses the opposite effect, namely the effect of manipulation of vibrotactile stimuli on sound perception, and that illustrates sensory integration in a quite natural context.

However, this has imposed strong constraints on the violinists who thus lost control on the production of the auditory and vibrotactile stimuli that are perceived, even if we took great care to place them in the most possible active situation regarding this production. Therefore, it remains unclear to which extent our results would hold in the real playing situation which leads not only to sensory integration but to sensory-motor integration: violinists control the sound production and thus the generation of the auditory and vibrotactile feedbacks that they perceive and that can modify, in return, the control within a complex loop. This study is nevertheless interesting as it illustrates, even if only in the particular context of this experiment, the influence of the level of violin vibrotactile feedback at the left hand on the perception 
and evaluation of some perceptual features of a violin, especially criteria related a priori only to the sound of the instrument. It thus sheds light on why correlations between sound perceived qualities and acoustical properties of violins have been so difficult to find and encourages researchers to extend their search to non-acoustical properties.

\section{ACKNOWLEDGMENTS}

The authors gratefully acknowledge the contribution of Frédéric Moisan as the professional violinist experimenter, and Quentin Vallet, Emmanuel Ponsot, Laurent Quartier, and Joël Frelat for their support and technical assistance. The luthiers Philippe Mitéran-Bigorgne, Frédéric Levi and Nicolas Perrin are acknowledged for their kind loan of violins. We are grateful to Vincent Hayward, Stephen McAdams, and Charles Spence for many helpful discussions and comments. This work was supported by the CNRS and UPMC (UMR 7190). I.W. was a recipient of a Ph.D. fellowship from UPMC-Ministry of Higher Education and Research.

${ }^{1}$ Below $600 \mathrm{~Hz}$, the typical vibrations levels in the necks at dynamics between $m f$ and $f f$ are roughly $30 \mathrm{~dB}(\mathrm{re} 1 \mu \mathrm{m} / \mathrm{N})$ above the detection threshold measured at the left hand (Wollman, 2013).

${ }^{2}$ Lecoutre, B., and Poitevineau, J. (1992). "Pac (software for analysis of comparisons): reference manual" (in French) (CISIA-CERESTA, Montreuil).

${ }^{3}$ Naturally they are conditional on the data at hand, but, for sake of brevity, the conditional notation is omitted.

${ }^{4}$ Although Table III is deductible from the two preceding comparisons (Table I and Table II), it is presented here for the convenience of the reader.

Askenfelt, A., and Jansson, E. V. (1992). "Vibration sensation in stringed instrument playing," Music Percept. 9(3), 311-350.

Calvert, G. A., Spence, C., and Stein, B. E., Eds. (2004). The Handbook of Multisensory Processes (MIT Press, Boston, MA), pp. 1-921.

Gillmeister, H., and Eimer, M. (2007). "Tactile enhancement of auditory detection and perceived loudness," Brain Res. 1160, 58-68.

Giordano, B. L., Avanzini, F., Wanderley, M. M., and Mcadams, S. (2010). "Multisensory integration in percussion performance," in Proceedings of the 10ème Congrès Français d'Acoustique, Lyon, pp. 1-6.

Hutchins, C. M. (1985). "Effect of an air-body coupling on the tone and playing qualities of violins," J. Catgut. Acoust. Soc. 44, 12-15.
Hsee, C. K., Loewenstein, G. F., Blount, S., and Bazerman, M. H. (1999). "Preference reversals between joint and separate evaluations of options: A review and theoretical analysis," Psychol. Bull. 125(5), 576.

Kayser, C., Petkov, C., Augath, M., and Logothetis, N. (2005). "Integration of touch and sound in auditory cortex," Neuron 48(2), 373-384.

Keane, M., and Dodd, G. (2011). "Subjective assessment of upright piano key vibrations," Acta Acust. Acust. 97(4), 708-713.

Lamoré, P. J. J., Muijser, H., and Keemink, C. J. (1986). "Envelope detection of amplitude-modulated high-frequency sinusoidal signals by skin mechanoreceptors," J. Acoust. Soc. Am. 79(4), 1082-1085.

Marshall, K. D. (1985). "Modal analysis of a violin,” J. Acoust. Soc. Am. 77(2), 695-709.

Marshall, K. D. (1986). "The musician and the vibrational behavior of a violin," J. Catgut. Acoust. Soc. 45, 28-33.

Musacchia, G., Strait, D., and Kraus, N. (2008). "Relationships between behavior, brainstem and cortical encoding of seen and heard speech in musicians and non-musicians," Hear. Res. 241(1), 34-42.

Rouanet, H., Bernard, J.-M., Bert, M.-C., Lecoutre, B., Lecoutre, M.-P., and Le Roux, B. (2000). New Ways in Statistical Methodology: From Significance Tests to Bayesian Inference, 2nd ed. (Peter Lang, Bern, Switzerland), pp. 1-276.

Saitis, C., Giordano, B. L., Fritz, C., and Scavone, G. (2012). "Perceptual evaluation of violins: A quantitative analysis of preference judgments by experienced players," J. Acoust. Soc. Am. 132(6), 4002-4012.

Stark, B., Carlstedt, T., Hallin, R. G., and Risling, M. (1998). "Distribution of human Pacinian corpuscles in the hand: A cadaver study," J. Hand Surg: Br. Eur. 23(3), 370-372.

Stein, B. E., and Meredith, M. A. (1993). The Merging of the Senses (MIT Press, Boston, MA), pp. 1-221.

Von Bekesy, G. (1959). "Similarities between hearing and skin sensations," Psychol. Rev. 66(1), 1.

Wollman, I. (2013). "Perception bimodale des violonistes en situation de jeu: influence des retours auditif et vibrotactile sur l'évaluation du violon" (Bimodal perception of violinist's play situation: Influence of auditory and vibrotactile feedback on the evaluation of the violin) $\mathrm{Ph} . \mathrm{D}$. dissertation, Paris VI University, France.

Wollman, I., Fritz, C., Poitevineau, J., and McAdams, S. (2013). "Violin quality evaluation: Examining the role of auditory and vibrotactile feedbacks," in Proceedings of the Stockholm Music Acoustics Conference, pp. 140-146.

Woodhouse, J. (1998). "The acoustics of 'A0-B0 mode matching' in the violin," Acta Acust. Acust. 84(5), 947-956.

Yau, J. M., Weber, A. I., and Bensmaia, S. J. (2010). "Separate mechanisms for audio-tactile pitch and loudness interactions," Front. Psychol. 1, 1-11.

Zampini, M., Guest, S., and Spence, C. (2003). "The role of auditory cues in modulating the perception of electric toothbrushes," J. Dent. Res. 82(11), 929-932.

Zampini, M., and Spence, C. (2004). "The role of auditory cues in modulating the perceived crispness and staleness of potato chips," J. Sens. Stud. 19(5), 347-363. 